\title{
Comparative Evaluation of Mixing Dynamies in USP Apparatus 2 using Standard USP Vessels and PEAK ${ }^{\text {TM Vessels }}$
}

\author{
Charles C. Collins and Renuka R. Nair \\ Graduate School of Pharmaceutical Sciences, \\ Duquesne University, Pittsburgh, PA
}

\begin{abstract}
Objective: To compare dissolution results for commercial formulations in USP Apparatus 2 using conventional USP vessels and PEAK ${ }^{\mathrm{TM}}$ vessels. Methods: Dissolution studies using commercially available disintegrating tablets of acetaminophen and naproxen sodium were conducted in USP Apparatus 2 using USP and PEAK ${ }^{\mathrm{TM}}$ vessels. The effect of four different RPMs $(25,50,75$ and 100) and three dissolution media (water, 1\% Tween 80 and phosphate buffer, pH 5.8 and 7.4 for acetaminophen and naproxen sodium, respectively) on the mixing dynamics in the two vessels were investigated. Both nondeaerated and deaerated media were employed in the dissolution studies. To confirm the differences in mixing, if any, is only due to the formation of a cone at the bottom of the USP vessel, dissolution studies on these tablets were also performed in USP Apparatus 1. Results: Significant differences ( $p$ $<0.05)$ were detected for up to 20 minutes in the release of naproxen sodium tablets at RPM 50,75 and 100 in nondeaerated and deaerated phosphate buffer ( $p H$ 7.4). However, no significant difference in mixing was seen at 25 RPM. Release
\end{abstract}

of the drug from naproxen sodium tablets showed significant differences $(p<0.05)$ in both nondeaerated and deaerated water and $1 \%$ Tween 80 with PEAK ${ }^{\mathrm{TM}}$ vessels showing greater release. Interestingly, no significant difference was detected in the release profile using USP Apparatus 1 indicating that the different mixing profile is in fact due to deposition of powder at the bottom of the USP vessel. Acetaminophen tablets showed differences in mixing in the two vessels only at RPM 25. There was no difference in the mixing of acetaminophen in the USP Apparatus 1 at 25 RPM. No difference was detected in water and $1 \%$ Tween 80 at 25 RPM or at higher RPMS of 50, 75 and 100 in phosphate buffer ( $p H$ 5.8). Conclusions: PEAK ${ }^{\mathrm{TM}}$ vessels show improved mixing when compared to USP vessels indicated by an increase in the release profile (and a decrease in variability) with the PEAK ${ }^{\mathrm{TM}}$ vessels. This is due to the shape of the vessel which belps to displace any cone effect at the bottom of the vessel. It is possible that tablets that may fail to meet USP dissolution criteria do so because of poor particle dispersion which can be overcome by the use of PEAK ${ }^{\mathrm{TM}}$ vessels.

\section{INTRODUETION}

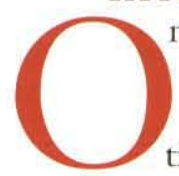

ne of the problems associated with USP Apparatus 2 is the formation of a cone of material at the bottom of the dissolution vessel. Dosage forms, especially ones with dense excipients, tend to form a cone at the bottom of the vessel giving rise to an unstirred region of drug material. This reduces the surface area of the dissolving material in contact with the dosage form and consequently affects the dissolution rate. Cone formation could be a problem predominantly with Apparatus 2, but may also happen in Apparatus 1 if the particles are released from the wire mesh basket. Earlier studies have established the influence of the geometry of the dissolu- tion vessel, especially the base curvature, on the flow of the dissolution media and the hydrodynamic mixing. Vessels with different bottom concavity lead to changes in hydrodynamic mixing within the dissolution media large enough to cause a significant change in the dissolution rate $(3,4,5)$. Recently VanKel introduced the PEAK ${ }^{\mathrm{TM}}$ dissolution vessel. The dimensions of this vessel are similar to the conventional USP vessel. The major difference is that this vessel has a small inverted cone at its base. In a study conducted by Dr. Arnold Beckett et al, PEAK $^{\mathrm{TM}}$ vessels were found to provide higher dissolution rates for calibrator tablets and NCDA\#2 prednisone tablets (6). In addition, it was also observed that the presence of dissolved air did not influence the dissolution results in the PEAK ${ }^{\mathrm{TM}}$ vessel due to effective displacement of the cone by the small indent at the base of the PEAK ${ }^{\mathrm{TM}}$ vessel. The present study was undertaken to determine if this vessel would influence dissolution studies on commercially available tablets. Since the difference in mixing between USP and PEAK ${ }^{\mathrm{TM}}$ vessels is assumed to be primarily due to the poorly mixed cone at the bottom of the USP vessel, it was hypothesized that as the speed increased, it would help to displace the cone. This would subsequently reduce the difference in mixing and dissolution rate in the two

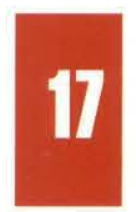




\section{Comparative Evaluation of Mixing Dynamics.... Eont.}

Table I. Dissolution data of acetaminophen disintegrating tablets in USP Apparatus 2 at various stirring speeds. [Phosphate Buffer, pH 5.8]

\begin{tabular}{|c|c|c|c|}
\hline RPM & Time (min) & \multicolumn{2}{|c|}{$\begin{array}{c}\text { Released } \\
\text { (Mean } \pm \text { S.D., } n=6 \text { ) }\end{array}$} \\
\hline & & USP & PE.AK ${ }^{\text {MM }}$ \\
\hline 25 & 5 & $15.81 \pm 1.13$ & $44.74 \pm 5.4$ \\
\hline & 10 & $30.15 \pm 2.02$ & $79.44 \pm 9.56$ \\
\hline & 15 & $39.56 \pm 2.44$ & $95.32 \pm 4.48$ \\
\hline & 20 & $46.38 \pm 2.84$ & \\
\hline & 25 & $51.68 \pm 2.94$ & \\
\hline & 30 & $55.51 \pm 3.66$ & \\
\hline 50 & 5 & $75.39 \pm 3.83$ & $81.87 \pm 0.86$ \\
\hline & 10 & $93.07 \pm 1.18$ & $94.97 \pm 0.58$ \\
\hline & 15 & $97.18 \pm 0.37$ & $97.33 \pm 1.43$ \\
\hline & 20 & $100.34 \pm 0.44$ & $100.53 \pm 0.88$ \\
\hline 75 & 5 & $84.54 \pm 1.98$ & $87.57 \pm 2.19$ \\
\hline & 10 & $96.74 \pm 1.21$ & $96.93 \pm 1.25$ \\
\hline & 15 & $100.49 \pm 0.67$ & $100.41 \pm 0.55$ \\
\hline 100 & 5 & $96.28 \pm 0.61$ & $96.21 \pm 0.77$ \\
\hline & 10 & $99.88 \pm 0.47$ & $99.61 \pm 0.46$ \\
\hline
\end{tabular}

Table II. Dissolution data of naproxen sodium tablets in USP Apparatus 2 at various stirring speeds. [Phosphate Buffer $\mathrm{pH} 7.4$ ]

\begin{tabular}{|c|c|c|c|}
\hline RPM & Time (min) & \% Released & (Mean \pm S.D., n-6) \\
\hline & & CSP & PE.AK $\mathbf{~}$ M \\
\hline $\mathbf{2 5}$ & 5 & $10.8 \pm 2.3$ & $9.58 \pm 2.5$ \\
\hline & 10 & $33.32 \pm 7.5$ & $35.84 \pm 3.34$ \\
\hline & 15 & $55.61 \pm 8.31$ & $55.77 \pm 4.46$ \\
\hline & 20 & $72.81 \pm 10.97$ & $71.81 \pm 5.33$ \\
\hline & 25 & $85.31 \pm 12.78$ & $83.41 \pm 4.18$ \\
\hline & 30 & $92.78 \pm 10.61$ & $94.82 \pm 5.98$ \\
\hline $\mathbf{5 0}$ & 5 & $19.12 \pm 8.36$ & $32.87 \pm 2.39$ \\
\hline & 10 & $51.53 \pm 15.06$ & $72.71 \pm 2.54$ \\
\hline & 15 & $74.68 \pm 17.24$ & $97.89 \pm 1.65$ \\
\hline & 20 & $88.59 \pm 14.93$ & $99.72 \pm 0.25$ \\
\hline & 25 & $93.3 \pm 9.83$ & \\
\hline & 30 & $96.04 \pm 6.91$ & \\
\hline $\mathbf{7 5}$ & 5 & $24.88 \pm 5.38$ & $44.42 \pm 4.57$ \\
\hline & 10 & $63.2 \pm 7.62$ & $83.51 \pm 2.00$ \\
\hline & 15 & $87.66 \pm 9.22$ & $101.27 \pm 0.68$ \\
\hline 100 & 5 & $28.33 \pm 7.55$ & $47.23 \pm 5.19$ \\
\hline & 10 & $65.29 \pm 10.1$ & $88.52 \pm 2.22$ \\
\hline & 15 & $87.68 \pm 7.93$ & $101.06 \pm 1.34$ \\
\hline
\end{tabular}

vessels. To check for consistency of results, dissolution studies were performed in three dissolution media. Also, since the difference in mixing was related to the shape of the vessel, it can be assumed that no difference would be seen with Apparatus 1. Dissolved gases have also been shown to affect the dissolution rate in USP vessels, either altering the flow pattern in the dissolution media or in some cases the boundary layer at the solid-liquid interface. In an attempt to investigate this effect, dissolution studies were performed in nondeaerated and deaerated media.

\section{METHODOLOGY}

Dissolution studies on commercially available disintegrating tablets of acetaminophen and naproxen sodium were conducted in USP Apparatus 2 using USP and PEAK ${ }^{\mathrm{TM}}$ vessels. Dissolution was performed at four different speeds: $25,50,75$ and 100 RPM. Three dissolution media [phosphate buffer $(\mathrm{pH}$ 5.8 and 7.4 for acetaminophen and naproxen sodium respectively), water, and $1 \%$ Tween 80 ] were used in the study. Both nondeaerated and deaerated media were used to investigate the influence of presence of air on the dissolution profile. Deaeration was achieved by sonicating the dissolution medium for 20 minutes. The temperature of the dissolution medium was maintained at $37( \pm 0.05)^{\circ} \mathrm{C}$. Dissolution of acetaminophen and naproxen sodium tablets were also conducted in USP Apparatus 1 at 50 RPM using phosphate buffer as the dissolution media with both the vessels. All analyses were performed by UV spectrophotometry. The detection wavelength varied with the drug: $243 \mathrm{~nm}$ for acetaminophen and $332 \mathrm{~nm}$ for naproxen. Standard curves were linear with $r^{2}$ values $\geq 0.999$. Significant differences in percent dissolution were carried out using a two-tailed ttest performed in Microsoft Excel software, Ver 5.0.

\section{RESULTS AND DISFISSIN}

1. Effect of RPM on the mixing dynamics in the two vessels: There was more than a twofold increase in the dissolution rate of acetaminophen in the PEAK ${ }^{T M}$ vessels compared to the
USP vessel at 25 RPM (Table I). The difference was observed throughout the sampling period. Almost $100 \%$ of the drug was released at the end of 15 min in the PEAK ${ }^{\text {TM }}$ vessel compared to only $40 \%$ release in the USP vessel. The percentage of drug released differed significantly $(\mathrm{p}<0.05)$ during the initial 10 minutes in the two type of vessels at 50 and 75 RPM with the PEAK $^{\mathrm{TM}}$ vessel showing a faster rate of dissolution. As the dissolution proceeded there appeared to be no difference in the release profile between the two vessels with all the drug being released by the end of 20 min. Similarly, no difference in dissolution was observed throughout the dissolution period between the two vessels at 100 RPM. Intensity of agitation affected the mixing in both the vessels but the effect was less pronounced in the PEAK ${ }^{\mathrm{TM}}$ vessel. At all stirring speeds, $100 \%$ of the drug was released in $20 \mathrm{~min}$ in the PEAK ${ }^{\mathrm{TM}}$ vessel. However, in the USP vessel the lower intensity of agitation at 25 RPM released only about $45 \%$ of the drug during the same time period. No significant difference in the release profile of naproxen sodium in the two vessels was seen at 25 RPM. Approximately $95 \%$ of the drug was released at the end of $30 \mathrm{~min}$ in both the vessels (Table II). The difference became apparent with increasing RPM. At 50 RPM, only $19 \%$ of the drug was released in the USP vessel within the first $5 \mathrm{~min}$ compared to a $32 \%$ release in the PEAK ${ }^{\mathrm{TM}}$ vessel. As expected, the difference between the two vessels decreased as dissolution proceeded. By the end of 20 minutes, in the PEAK ${ }^{\mathrm{TM}}$ vessel 
$100 \%$ of the drug was released compared to $75 \%$ release in the USP vessels. In addition, there appeared to be a greater variability in the dissolution data for the USP vessel. The variability ranged from $8-14 \%$ in the USP vessel compared to only $0.2-2 \%$ in the PEAK ${ }^{\mathrm{TM}}$ vessel. Similar differences in the dissolution rate and variability between the two vessels were observed at 75 and 100 RPM. During the dissolution process, the acetaminophen tablets disintegrated into granules. At the low stirring speed of 25 RPM, these granules settled at the bottom of both vessels which led to slower mixing. The difference between the two vessels was due to the small indentation present in the PEAK $^{\mathrm{TM}}$ vessel. As the stirring speed was increased, the granules, being light in nature, tended to disperse throughout the medium in both the vessels rather than settle at the base. Hence, no difference in mixing was seen at the higher rates of agitation. However, naproxen sodium tablets disintegrated into small particles. These particles tended to form a compact mass in both vessels. At 25 RPM, the intensity of agitation was too low and the mass of particles were too compact to be quickly displaced in either of the vessels. Hence, no difference in mixing was observed. However, as the stirring speed was increased, it tended to displace the cone more effectively in the PEAK $^{\mathrm{TM}}$ vessel by virtue of the indent at the bottom of the vessel. This, however, was not the case with the USP vessel, thereby leading to a greater dissolution in the PEAK ${ }^{\mathrm{TM}}$ vessels. The compact mass at the base of the
USP vessel also gave rise to greater variability as evident by the standard deviations.

\section{Effect of dissolution media:}

\section{Acetaminophen}

disintegrating tablets

As seen in Table III, a small difference of about $4 \%$ was seen in the first 5 $\min$ in the percent release of acetaminophen when the dissolution was performed in phosphate buffer $\mathrm{pH}$ 5.8. When the dissolution media was replaced by water this difference increased to $10 \%$ with the PEAK ${ }^{\text {TM }}$ vessels showing higher dissolution rate. The higher solubility of acetaminophen in Tween 80 resulted in no observed difference between the two vessels.

\section{Naproxen}

\section{sodium tablets}

Differences in mixing profile were seen for all dissolution media in the case of naproxen sodium tablets (Table IV). Approximately $50 \%$ of the drug was released in the PEAK $^{\mathrm{TM}}$ vessel within the initial 5 minutes compared to only $30 \%$ release in the USP vessel with water as the dissolution medium. A significant difference $(p<0.05)$ was observed for the Tween 80 solution only during the initial 5 minutes. This is because the surfactant reduces the interfacial tension between the solid cone and the surrounding media in the USP vessel and narrows the differences in release rate between
Table III. Dissolution data of acetaminophen disintegrating tablets in USP Apparatus 2 using various dissolution media.

\begin{tabular}{|c|c|c|c|c|c|c|}
\hline Time & \multicolumn{5}{|c|}{$\begin{array}{c}\text { \% Released - 50 RPM } \\
\text { (Mean } \pm \text { S.D., } \mathrm{n}=6 \text { ) }\end{array}$} \\
\hline (min) & Phosphate buffer $(\mathrm{pH} 5.8$ ) & \multicolumn{2}{|c|}{ Water } & \multicolumn{2}{c|}{$1 \%$ Tween 80 } \\
\hline & USP & PEAK & USP & PEAK & USP & PEAK \\
\hline 5 & $75.39 \pm 3.83$ & $81.87 \pm 0.86$ & $80.15 \pm 5.63$ & $92.4 \pm 3.15$ & $95.35 \pm 3.68$ & $97.65 \pm 2.16$ \\
10 & $93.07 \pm 1.18$ & $94.97 \pm 0.58$ & $97.51 \pm 2.17$ & $100.76 \pm 1.5$ & $100.13 \pm 1.7$ & $98.32 \pm 1.14$ \\
15 & $97.18 \pm 0.37$ & $97.33 \pm 1.43$ & $100.17 \pm 0.28$ & & & \\
20 & $100.34 \pm 0.44$ & $100.55 \pm 0.88$ & & & & \\
\hline
\end{tabular}

Table IV. Dissolution data of naproxen sodium tablets in USP Apparatus 2 in various dissolution media

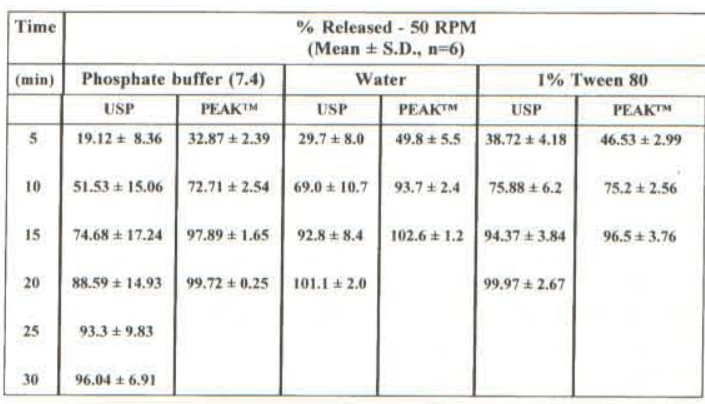

Table V. Dissolution data for acetaminophen disintegrating tablets in USP Apparatus 1 and 2 using USP and PEAK ${ }^{\mathrm{TM}}$ vessels. [Phosphate Buffer pH 5.8]

\begin{tabular}{|c|c|c|c|c|}
\hline Time & \multicolumn{4}{|c|}{$\begin{array}{c}\text { \% Released }-50 \text { RPM } \\
\text { (Mean } \pm \text { S.D., } \mathrm{n}=6 \text { ) }\end{array}$} \\
\hline (min) & \multicolumn{2}{|c|}{ Apparatus 1 } & \multicolumn{2}{c|}{ Apparatus 2 } \\
\hline & USP & PEAK ${ }^{\mathrm{TM}}$ & USP & PEAK ${ }^{\mathrm{TM}}$ \\
\hline 5 & $44.66 \pm 4.22$ & $45.2 \pm 6.05$ & $75.39 \pm 3.83$ & $81.87 \pm 0.86$ \\
10 & $78.39 \pm 6.57$ & $79.74 \pm 5.61$ & $93.07 \pm 1.18$ & $94.97 \pm 0.58$ \\
15 & $91.88 \pm 5.45$ & $92.45 \pm 4.54$ & $97.18 \pm 0.37$ & $97.33 \pm 1.43$ \\
20 & $97.77 \pm 4.23$ & $96.69 \pm 3.42$ & $100.34 \pm 0.44$ & $100.55 \pm 0.88$ \\
25 & $100.27 \pm 0.8$ & $100.68 \pm 0.71$ & & \\
\hline
\end{tabular}

the USP and PEAK ${ }^{\mathrm{TM}}$ vessels. However, the difference is too small to have any practical implications.

\section{Testing Devices}

Apparatus 1 and Apparatus 2 When performing the dissolution of acetaminophen in Apparatus 2 at 50 RPM, a significant difference $(\mathrm{p}<0.05)$ in mixing was observed in the initial 10 minutes. However, in Apparatus 1 there was no significant difference (Table V). In the case 


\section{Comparative Evaluation of Mixing Dynamics....tont.}

Table VI. Dissolution data for naproxen sodium disintegrating tablets in
Apparatus 1 and 2 using USP and PEAKTM vessel.
[Phosphate Buffer pH 7.4]

Table VII. Dissolution data of acetaminophen disintegrating tablets in USP Apparatus 2 using USP and PEAK ${ }^{\mathrm{TM}}$ vessels using nondeaerated and deaerated phosphate buffer (pH 5.8).

\begin{tabular}{|c|c|c|c|c|}
\hline \multirow{2}{*}{ Time (min) } & \multicolumn{4}{|c|}{$\begin{array}{c}\text { \% Released } \\
\text { (Mean } \pm \text { S.D., } \mathrm{n}=6 \text { ) }\end{array}$} \\
\cline { 2 - 5 } & \multicolumn{2}{|c|}{ USP } & \multicolumn{2}{c|}{ PEAK ${ }^{\mathrm{M}}$} \\
\hline 25 RPM & Deaerated & Nondeaerated & Deaerated & Nondeaerated \\
\hline 5 & $15.81 \pm 1.13$ & $29.41 \pm 2.44$ & $44.74 \pm 5.4$ & $55.39 \pm 5.96$ \\
\hline 10 & $30.15 \pm 2.02$ & $45.80 \pm 4.11$ & $79.44 \pm 9.56$ & $83.14 \pm 4.28$ \\
\hline 15 & $39.56 \pm 2.44$ & $55.91 \pm 5.94$ & $95.32 \pm 4.48$ & $97.20 \pm 3.6$ \\
\hline 20 & $46.38 \pm 2.84$ & $62.95 \pm 7.29$ & & \\
\hline 25 & $51.68 \pm 2.94$ & $66.78 \pm 8.73$ & & \\
\hline 30 & $55.51 \pm 3.66$ & $73.31 \pm 8.95$ & & \\
\hline 50 RPM & & & & \\
\hline 5 & $75.39 \pm 3.83$ & $85.82 \pm 5.18$ & $81.87 \pm 0.86$ & $96.89 \pm 3.92$ \\
\hline 10 & $93.07 \pm 1.18$ & $96.87 \pm 1.85$ & $94.97 \pm 0.58$ & \\
\hline 15 & $97.18 \pm 0.37$ & & $97.33 \pm 1.43$ & \\
\hline 20 & $100.34 \pm 0.44$ & & $100.53 \pm 0.88$ & \\
\hline \multicolumn{5}{|l|}{} \\
\hline
\end{tabular}

Table VIII. Dissolution data of naproxen sodium in USP Apparatus 2 using USP and PEAK ${ }^{\mathrm{M} M}$ vessels using nondeaerated and deaerated phosphate buffer ( $\left.\mathrm{pH} 7.4\right)$.

\begin{tabular}{|c|c|c|c|c|}
\hline \multirow[t]{3}{*}{ Time (min) } & \multicolumn{2}{|c|}{$\%$ Releused } & \multicolumn{2}{|c|}{ (Mean \pm S.D., n-6) } \\
\hline & \multicolumn{2}{|c|}{ USP } & \multicolumn{2}{|c|}{ PEAK'TM } \\
\hline & Deaerated & Noadeacrated & Deaerated & Nondeaerated \\
\hline S0 RPM & + & 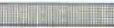 & 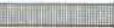 & - \\
\hline 5 & $19.12 \pm 8.36$ & $15.6 \pm 5.94$ & $32.87 \pm 2.39$ & $32.18 \pm 3.99$ \\
\hline 10 & $51.53 \pm 15.06$ & $46.06 \pm 8.56$ & $72.71 \pm 2.54$ & $70.24 \pm 6.9$ \\
\hline 15 & $74.68 \pm 17.24$ & $68.22 \pm 9.81$ & $97.89 \pm 1.65$ & $94.93 \pm 5.54$ \\
\hline 20 & $88.59 \pm 14.93$ & $74.89 \pm 12.85$ & $99.72 \pm 0.25$ & \\
\hline 25 & $93.3 \neq 9.83$ & $92.72 \pm 6.66$ & & \\
\hline 30 & $96.04 \pm 6.91$ & $96.83 \pm 4.58$ & & \\
\hline \multicolumn{5}{|l|}{75 RPM } \\
\hline 5 & $24.88 \pm 5.38$ & $23.95 \pm 4.32$ & $44.42 \pm 4.57$ & $46.64 \pm 3.05$ \\
\hline 10 & $63.2 \pm 7.62$ & $55.43 \pm 7.95$ & $83.51 \pm 2.00$ & $87.92 \pm 1.85$ \\
\hline 15 & $87.66 \pm 9.22$ & $78.38 \pm 9.06$ & $101.27 \pm 0.68$ & $101.88 \pm 0.66$ \\
\hline 20 & $101.15 \pm 4.85$ & $92.45 \pm 7.49$ & & \\
\hline 25 & & $99.25 \pm 4.48$ & & \\
\hline \multicolumn{5}{|l|}{$100 \mathrm{RPM}$} \\
\hline 5 & $28.33 \div 7.55$ & $29.88 \pm 5.25$ & $47.23 \pm 5.19$ & $49.93 \pm 3.31$ \\
\hline 10 & $65.29 \pm 10.1$ & $66.67 \pm 6.52$ & $88.52 \pm 2.22$ & $92.73 \pm 2.06$ \\
\hline 15 & $87.68 \pm 7.93$ & $91.08 \pm 5.7$ & $101.06 \pm 1.34$ & $99.33 \pm 1.1$ \\
\hline 20 & $98.02 \pm 3.18$ & $99.28 \div 0.78$ & & \\
\hline
\end{tabular}

of naproxen sodium tablets, for Apparatus 2, a very large difference of $12-20 \%$ was detected between the two vessels throughout the sampling period. However, with Apparatus 1, no significant difference was seen in the two vessels (Table
VI). Since the naproxen sodium tablet disintegrates into particles, some of the particles fell through the basket and settled at the bottom. This may account for the slight difference observed during the initial 10 minutes. In Apparatus 1 , any difference in mixing could be due to the mesh size. Unlike Apparatus 2, the curvature of the dissolution flask should have minimal impact on the dissolution rate since the dosage form is placed inside the basket. Since similar mixing profiles were observed for Apparatus 1 and dissimilar mixing profiles were observed for Apparatus 2 for the same dosage forms and dissolution test parameters the hypothesis that the shape of the vessel is a significant contributor to the differences is confirmed. This again confirms that the formation of a cone in the USP vessel can result in non-formulation related decrease in dissolution rate.

\section{Influence of Nondeaer- ation/Deaeration}

There was a significant difference $(\mathrm{p}<0.05)$ in the amount of acetaminophen released in the nondeaerated/deaerated media in both USP and PEAK ${ }^{\mathrm{TM}}$ vessels (Table VII). The amount of drug released was higher in the nondeaerated media compared to the deaerated media in both the vessels. There was also a significant difference in the release profile of acetaminophen in the two vessels in nondeaerated media.
Approximately $60 \%$ of the drug was released in the nondeaerated media using USP vessels at the end of 20 minutes compared to $100 \%$ release with the PEAK ${ }^{\mathrm{TM}}$ vessels. A significant difference $(p<0.05)$ in the mixing profile due to nondeaeration was also observed at 50 RPM in the two vessels. There was no difference in the dissolution profile of naproxen sodium tablets in nondeaerated/ deaerated media in either of the two vessels (Table VIII). For the same hydrodynamic reasons discussed in section two (above), dissolution rate differences for naproxen sodium were observed between the PEAK $^{\mathrm{TM}}$ and USP vessels in nondeaerated media at 50,75 and 100 RPM (Table VIII).

\section{RONBLIUSIONS}

The PEAK ${ }^{\mathrm{TM}}$ vessels eliminate the formation of a cone of materials in Apparatus 2 often observed with certain kinds of dosage forms. This was evident from visual observation of the vessel during dissolution study and also from the dissolution results. The PEAK ${ }^{\mathrm{TM}}$ vessels showed a higher release rate with both the commercially available disintegrating tablets employed in this study. Contrary to previous studies, the results from this study indicated that stirring speed and the presence of air can influence the amount of drug released in both vessels. PEAK ${ }^{\mathrm{TM}}$ vessels, however, provide an effective way to improve the mixing hydrodynamics in the dissolution vessel and reduce the variability typically observed for conventional USP vessels.

See References...continued next page 


\section{Produrt Quality Research initiative Holds Initial Meeting \\ by Bavid Pang, Ph.n., Aaps Director of Seientific Attairs}

At the Initial Meeting of the Product Quality Research Initiative (PQRI), held February 10-11, 1998, in Washington, DC, Gordon Amidon, Ph.D., AAPS President, and Larry Augsburger, Ph.D., AAPS President-Elect, presented results from their respective research projects, which could be considered as building blocks in the estab lishment of PQRI. In addition, Dr. Amidon delineated the roles that AAPS will undertake for this initiative. $P Q R I$ is an initiative for the Food and Drug Administration (FDA), pharmaceutical companies and universities to join together to provide opportunities to conduct scientific research projects designed to maintain product quality during drug development and evaluation. About 140 representatives from FDA, academia and industry participated in the meeting. The vigorous discussions and suggestions by the participants resulted in the formulation of clear objectives and research projects for PQRI.

PQRI projects will be performed in anticipation of coming improvernents in the specification requirements in testing procedures for product quality. It is hoped that the pharmaceutical industry will benefit from some change in the regulatory process in the form of shortening the drug application process and facilitating the approwal process within FDA. Some of the projects being considered fall into areas of drug substance, drug products, biopharmaceutics and science management. It is anticipated that the research projects could be funded by grants from pharmaceutical companies or other sources. Two pharmaceutical companies have pledged finan cial support for the continuation of the projects.

AAPS is working in collaboration with FOA to formalize and man. age PQRI. The roles for MPS in this initiative include: providing sclentific expertise and a neutral environment; providing administrative functions for PQRl; dispersing PQRI funds: organizing AAPS workshops to discuss findings of the research projects and conveying the resulting recommendations to FDA; and, coordinating PQRl-relat: ed training.

Other participating organizations in PQRI include Pharmaceutical Research and Manufacturers of America (PhRMA), Generic Pharma ceutical Industry Association \{GPIA\}, National Association of Phar: maceutical Manufacturers (NAPM). Parenteral Drug Association (PDA). Nonprescription Drug Manufacturers Association'(NDMA) and National Pharmaceutical Alliance (NPA).

References...comtmised from proviones page

\section{REFERENGES}

1. U.M.Banakar. In Pharmatcentical Dissolution Tecbnology, Marcel Dekker, Inc. NY (1991) pp 97. R. J. Withey and A. J. Bowker. Agitation of the solvent in tablet dissolution studies - a flow visualization technique. 24:345-351 (1972).

2.D.C.Cox, C. E. Wells, W. B. Furman, T. S. Savage and A. C. King. Systematic error associated with Apparatus 2 of the

\section{Sotax Awards}

SOTAX AG celebrates its 25 th anniversary in 1998 and to mark this occasion, it has decided to set up an award for scientists in the field of biopharmaceutics. The award will be directed towards scientists who are involved in investigations using the flow-through system. The scientific and technical investigation may be predominantly focused on studies concerning different types of flow-through cells and dosage forms (e.g. immediate and concolled/modified release tablets/capsules, scmi-solid dosage forms and parenterals such as implants, microparticles and suspensions). All studies performed using the flow-through method for research, development and quality control purposes are of interest. General investigations into the qualification/calibration and validation of the test procedure itself will also be considered for the award.

The scientific results may be presented in the form of publications or doctoral theses which were published or completed during the last 3 years. Scientific and/or technical documents should be sent to SO'TAX AG by September 30, 1998.

The papers sill be judget by an international committee headed by Prof. Dr. II. Möller and the following prizes will be awarded:

$\begin{array}{ll}\text { Ist Prize } & 3000 \text { Swiss francs } \\ \text { 2nd Prize } & 2000 \text { Swiss francs } \\ \text { 3rd Prize } & 1000 \text { Swiss francs }\end{array}$

SOTAX AG BASEL.

Binningestr 106

CII 4123 Allschwil/Switzerland

Phone 061/4875454

Fax 061/481 1331

Email Sotax@swissonline.com

Apparatus USP-NF requirements. 7. Pbarm. Sci. 61(10): [638-1640 (1972).

5. A. II. Beckett and G. S. Kurs. Improved Hydrodynamics for USP Apparatus 2. Discolution Tecbuologies, 3(2) May 1996.

\section{ACKNOWLEDGMENTS}

We gratefully acknowledge VanKel Industries for the donation of the standard USP and Peak dissolution vessels.
B. Schwart\%. Variations in dissolution data using an 